\title{
Immuno-oncology for esophageal cancer
}

\author{
Shun Yamamoto ${ }^{1}$ (iD) \& Ken Kato*,1(i) \\ ${ }^{1}$ Department of Head \& Neck Medical Oncology/Department of Gastrointestinal Medical Oncology, National Cancer Center \\ Hospital, Tokyo, Japan \\ *Author for correspondence: Tel.: +813 3542 2511; Fax: +813 3542 3815; kenkato@ncc.go.jp
}

Esophageal cancer (EC) is the seventh most common cancer, with a poor prognosis for metastatic EC patients and limited effective drugs for treatment. Nivolumab and pembrolizumab, monoclonal antibodies that inhibit interactions of PD-1 and its ligand (PD-L1), which induce lymphocyte activation, have antitumor activity. The ATTRACTION-3 trial compared nivolumab with taxane after first-line chemotherapy and demonstrated superior overall survival (OS) for esophageal squamous cell carcinoma. The KEYNOTE-181 trial that compared pembrolizumab with chemotherapy demonstrated superior OS for EC with a PD-L1 combined positive score $\geq 10$. Trials to evaluate efficacy of combined cytotoxic agents and immune checkpoint inhibitors $(\mathrm{ICl})$ as first-line chemotherapies, ICl-containing chemoradiotherapy, and pre/postoperative chemotherapy are ongoing and might provide important data to improve clinical outcomes.

First draft submitted: 27 May 2020; Accepted for publication: 10 July 2020; Published online: 11 August 2020

Keywords: atezolizumab • esophageal cancer • immune checkpoint inhibitor • nivolumab • pembrolizumab • tislelizumab

\section{Introduction}

Esophageal cancer (EC) is the seventh most common cancer and the sixth leading cause of cancer deaths worldwide [1]. Esophageal cancer can be broadly divided into two major histologic subtypes: esophageal adenocarcinoma (EAC) and esophageal squamous cell carcinoma (ESCC), the most frequent histologic subtype accounting for $87 \%$ of all esophageal cancers worldwide [2]. Regional and cultural differences exist with regard to the dominant histological subtype. Although the incidence of ESCC patients has shown an increasing trend in Asian countries, ESCC has shown a decreasing trend in North America and Europe [1]. In contrast, the incidence of EAC patients is increasing steadily in Western countries [1].

The major risk factors for ESCC and EAC are different. Smoking and alcohol consumption are the main risk factors for ESCC, and chronic gastroesophageal reflux disease is the main risk factor for EAC [3,4].

In general, EC is a highly aggressive malignant disease, most EC patients are diagnosed at an advanced stage, and the prognosis of metastatic or recurrent EC patients is extremely poor with a median overall survival (OS) of 4 to 6 months based on data from the Surveillance, Epidemiology, and End Results registry [5]. In addition, ESCC was associated with poorer survival compared with EAC [5].

The standard treatments for EC patients are determined using the clinical Tumour-Node-Metastasis (TNM) stage. For patients with metastatic or recurrent EC without indication for curative treatments such as surgery and definitive chemoradiotherapy, systemic chemotherapy is an important treatment aiming to palliate symptoms and improve survival [6-8]. Although no randomized controlled trials (RCTs) have shown additional survival benefit of palliative chemotherapy compared with the best supportive care as first-line treatment, doublet chemotherapy consisting of platinum and fluoropyrimidine (e.g., cisplatin and 5-FU [CF], oxaliplatin, and 5-FU plus leucovorin: FOLFOX) is recognized as the first-line standard chemotherapy for metastatic or recurrent EC patients [6-10]. After refractory or intolerance to first-line chemotherapy, taxane [11,12] or irinotecan [6] monotherapy as salvage line chemotherapy is used for metastatic or recurrent EC patients. A meta-analysis showed clinical benefit of these treatments compared with cytotoxic agents with best supportive care [13]. However, there have been no RCTs on the efficacy and safety of palliative chemotherapy for metastatic or recurrent EC patients. 
For patients with unresectable locally advanced EC without metastatic lesions, definitive chemoradiotherapy is considered the standard treatment. In particular, the combination treatment of CF regimen and 50.4- to 60.0-Gy radiation [14,15] and combination treatment of FOLFOX regimen and 50-Gy radiation [16] are commonly performed as standard treatments.

For patients with resectable locally advanced EC, preoperative chemotherapy consisting of CF $[17,18]$ or chemoradiotherapy consisting of carboplatin and paclitaxel plus radiation at a dose of $41.4 \mathrm{~Gy}$ [19] followed by surgery has been established as the standard treatment based on RCTs.

Therefore, there are two major unmet clinical needs for the treatment for EC patients. First, there are few effective cytotoxic agents for metastatic or recurrent EC patients; thus, novel agents and strategies are required to improve clinical outcomes. Second, the efficacy of definitive chemoradiotherapy or preoperative therapy for patients with locally advanced EC is limited. The median OS of locally advanced EC patients who received definitive chemoradiotherapy was 17 to 20 months [16], and the 5-year OS rate of resectable locally advanced EC patients who received the standard strategy was 47 to $55 \%[17,19]$.

In general, tumor cells escape elimination by cytotoxic $\mathrm{T}$ cells and thus are known to suppress the effector function of tumor-specific $\mathrm{T}$ cells through immune checkpoint molecules and regulatory $\mathrm{T}$ cells. Therefore, the elimination of tumor cells by cytotoxic $\mathrm{T}$ cells is thought to occur by suppressing the immune checkpoint molecule, which is a co-suppressor represented by PD-1 and cytotoxic CTLA-4.

For these situations, immune checkpoint inhibitors (ICI) with new mechanisms of action have been developed for various types of cancer [20-32]. For example, PD-1, a negative regulatory molecule expressed by activated lymphocytes, binds to its ligands, PD-L1 and 2 (PD-L2), resulting in the downregulation of lymphocyte activation and suppression of antitumor immune responses. Anti-PD-1 antibody and anti-PD-L1 antibody inhibit the interaction of PD-1 and PD-L1/PD-L2, preventing the inhibition of lymphocyte activation, and therefore showing antitumor activity [33].

Nivolumab, an anti-PD-1 antibody, was developed for metastatic or recurrent ESCC and has shown significant efficacy and safety based on results of the Phase II ATTRACTION-1 trial [34] and the randomized Phase III ATTRACTION-3 trial [35]. Pembrolizumab, another anti-PD-1 antibody, was developed for metastatic or recurrent EC with combined positive score (CPS) $\geq 10$ and showed significant efficacy and safety based on the results of the Phase II KEYNOTE-180 trial [36] and the randomized Phase III KEYNOTE-181 trial [37].

ICIs were developed for the salvage line monotherapy of metastatic or recurrent EC patients, and the recent development of combination treatments consisting of ICI plus cytotoxic agents or radiation has been promoted. In this review, we outline the status of the development of ICI-containing treatments for EC patients in each clinical setting.

\section{ICI treatment development for metastatic or recurrent EC}

In 2019, two new ICIs, nivolumab and pembrolizumab, showed clinical benefit as salvage-line chemotherapy for metastatic or recurrent EC patients.

Nivolumab is a novel human monoclonal antibody that targets PD-1. The Phase II ATTRACTION-1 trial showed the promising efficacy and safety of nivolumab. Sixty-five patients with metastatic ESCC refractory or intolerant to standard chemotherapies were included in the trial. The objective response rate, median progression-free survival (PFS) and median OS were 17\% (95\% CI: 10-28\%), 1.51 months (95\% CI: 1.41-2.83 months) and 10.78 months (95\% CI: 7.39-13.34 months), respectively. The most frequent grade 3-4 adverse events were lung infection (3\%) and dehydration (3\%), and there were no deaths related to nivolumab [34].

After the promising results of the Phase II trial, the randomized Phase III ATTRACTION-3 trial that compared nivolumab with paclitaxel or docetaxel after refractory or intolerant to one prior fluoropyrimidine/platinum-based chemotherapy for metastatic ESCC patients mostly from Asia showed superior OS (10.9 months vs 8.4 months, hazard ratio [HR]: $0.77 ; 95 \%$ CI: $0.62-0.96 ; \mathrm{p}=0.019)$ and equivalent objective response rates (19 vs $22 \%)$. In the nivolumab arm, the most frequent serious adverse events were pyrexia (2\%) and intestinal lung disease (2\%), and there were two deaths related to nivolumab (interstitial lung disease and pneumonitis) [35]. On the basis of these results, the US Food and Drug Administration (FDA) approved nivolumab monotherapy as a treatment for advanced ESCC patients after prior fluoropyrimidine- and platinum-based chemotherapy regardless of PD-L1 expression on June 10, 2020. Nivolumab has become the standard second-line chemotherapy for metastatic or recurrent ESCC patients. 


\begin{tabular}{|c|c|c|c|c|c|c|c|}
\hline Clinical trial & Phase & Patients (n) & Study treatment & ORR (\%) & PFS (m) & OS (m) & Ref. \\
\hline \multicolumn{8}{|c|}{ Later-line chemotherapy } \\
\hline ATTRACTION-1 & II & 65 & Nivolumab monotherapy & 17 & 1.5 & 10.8 & [34] \\
\hline ATTRACTION-3 & III & 419 & Nivolumab monotherapy vs cytotoxic chemotherapy & 19 & 1.7 & 10.9 & [35] \\
\hline KEYNOTE-180 & II & 121 & Pembrolizumab monotherapy & 9.9 & 2.0 & 5.8 & [37] \\
\hline KEYNOTE-181 & III & 628 & Pembrolizumab monotherapy vs cytotoxic chemotherapy & 13.1 & 2.1 & 7.1 & [38] \\
\hline ESCORT & III & 457 & Camrelizumab monotherapy vs cytotoxic chemotherapy & 20.2 & 1.9 & 8.3 & [40] \\
\hline RATIONALE-302 & III & Before analysis & Tislelizumab vs cytotoxic chemotherapy & NA & NA & NA & \\
\hline \multicolumn{8}{|c|}{ First-line chemotherapy } \\
\hline CheckMate-648 & III & Before analysis & Nivolumab + ipilimumab vs $\mathrm{CF}+$ nivolumab vs $\mathrm{CF}$ & NA & NA & NA & \\
\hline KEYNOTE-590 & III & Before analysis & 5 -FU + platinum \pm pembrolizumab & NA & NA & NA & [39] \\
\hline RATIONALE-306 & III & Recruiting & $5-F U / P T X+$ platinum \pm tislelizumab & NA & NA & NA & \\
\hline
\end{tabular}

Ipilimumab is a human monoclonal antibody that targets CTLA-4, a negative regulatory molecule expressed by T cells, binds to B7 ligands, resulting in the downregulation of lymphocyte activation and suppression of antitumor immune responses. Therefore, anti-CTLA- 4 antibody shows antitumor activity by preventing the inhabitation of lymphocyte activation and doublet ICI therapy consisting of anti-PD-1 antibody, which has a different mechanism of action; thus, the anti-CTLA-4 antibody might show greater antitumor efficacy than ICI monotherapy. The CheckMate-032 Phase I/II trial evaluating safety and efficacy of doublet therapy consisting of ipilimumab and nivolumab for patients with previously treated metastatic esophageal or esophagogastric, gastric adenocarcinoma showed that objective response rates were 24\% (95\% CI: 13-39\%) in nivolumab $1 \mathrm{mg} / \mathrm{kg}$ plus ipilimumab $3 \mathrm{mg} / \mathrm{kg}$ arm and $8 \%$ (95\% CI: 2-19\%) in nivolumab $3 \mathrm{mg} / \mathrm{kg}$ plus ipilimumab $1 \mathrm{mg} / \mathrm{kg}$ arm [36].

In addition to these trials for previously treated ESCC and EAC patients, a Phase III CheckMate-648 trial to compare nivolumab plus $\mathrm{CF}$ or nivolumab plus ipilimumab with $\mathrm{CF}$ as a first-line chemotherapy for untreated patients with metastatic or recurrent ESCC (NCT03143153) is ongoing Table 1.

Pembrolizumab is another human monoclonal antibody that targets PD-1. A Phase II KEYNOTE-180 trial to evaluate the efficacy and safety of pembrolizumab was conducted. It included 121 patients, of which $63(52 \%)$ had ESCC and 58 (47.9\%) had PD-L1 positive tumors. PD-L1 positivity was defined as CPS (the ratio of the number of PD-L1 positive cells divided by the total number of tumor cells, multiplied by 100) $\geq 10$ that was evaluated by immunohistochemistry. The objective response rate was $9.9 \%$ (95\% CI: $5.2-16.7)$ in all patients, $14.3 \%$ (95\% CI: 6.7-25.4) in ESCC patients, 5.3\% (95\% CI: 1.1-14.4) in EAC patients, 13.8\% (95\% CI: 6.1-25.4) in patients with CPS $\geq 10$ and $6.3 \%$ (95\% CI: 1.8-15.5) in patients with CPS $<10$; the median duration of response was not reached. The median PFS was 2.0 months (95\% CI: 1.9-2.1) in all patients, 2.1 months (95\% CI: $2.0-2.4)$ in ESCC patients, 1.9 months (95\% CI: 1.8-2.0) in EAC patients, 2.0 months (95\% CI: 1.9-2.2) in patients with CPS $\geq 10$ and 2.0 months (95\% CI: 1.9-2.1) in patients with CPS $<10$. The median OS was 5.8 months (95\% CI: 4.5-7.2) in all patients, 6.8 months (95\% CI: 5.4-8.9) in ESCC patients, 5.4 months (95\% CI: 3.2-6.3) in EAC patients, 3.9 months (95\% CI: 4.4-9.3) in patients with CPS $\geq 10$ and 5.4 months (95\% CI: 3.9-6.3) in patients with CPS $<10$. In the Phase II trial, frequent grade 3-4 adverse events related to pembrolizumab included type 1 diabetes mellitus $(3 \%)$ and pneumonitis $(2 \%)$ and one patient had a grade 5 treatment-related adverse event (pneumonitis) [37].

After the KEYNOTE-180 trial, the Phase III KEYNOTE 181 trial comparing pembrolizumab with an investigator's choice regimen (paclitaxel or docetaxel, irinotecan) for metastatic or recurrent EC in second-line treatment was conducted. The primary endpoints were OS of patients with PD-L1 CPS $\geq 10$ or with ESCC, in all patients. Because of multiple primary endpoints, the significance levels were alpha $0.9 \%(\mathrm{p} \leq 0.0085)$ in patients with PD-L1 CPS $\geq 10$, alpha $0.8 \%(\mathrm{p} \leq 0.0077)$ in patients with ESCC and alpha $0.8 \%(\mathrm{p} \leq 0.0077)$ in all patients, respectively. All 628 patients were randomly assigned 1:1 to pembrolizumab or the investigator's choice regimen. In $222 \mathrm{EC}$ patients with PD-L1 CPS $\geq 10$, pembrolizumab monotherapy significantly improved the OS (9.3 vs 6.7 months; HR: 0.69; 95\% CI: 0.52-0.93; $p=0.0074)$ compared with cytotoxic chemotherapy. However, pembrolizumab did not demonstrate superior OS compared with cytotoxic chemotherapy in 401 patients with ESCC (8.2 vs 7.1 months; HR: 0.78; 95\% CI: 0.63-0.96; $\mathrm{p}=0.0095)$ and in all 628 patients (7.1 vs 7.1 months; 
HR: 0.89; 95\% CI: 0.75-1.05; $\mathrm{p}=0.0560$ ). PFS in $222 \mathrm{EC}$ patients with PD-L1 CPS $\geq 10$ was 2.6 months for pembrolizumab and 3.0 months for the cytotoxic chemotherapy regimen, and the objective response rate in EC patients with PD-L1 CPS $\geq 10$ was $21.5 \%$ for pembrolizumab and $6.1 \%$ for the cytotoxic chemotherapy regimen $(p=0.0006)$. PFS in 401 ESCC patients was 2.2 months for pembrolizumab and 3.1 months for cytotoxic chemotherapy regimen, and the objective response rate in ESCC patients was $16.7 \%$ for pembrolizumab and $7.4 \%$ for cytotoxic chemotherapy regimen $(\mathrm{p}=0.0022)$. PFS in all 628 ESCC patients was 2.1 months for pembrolizumab and 3.4 months for cytotoxic chemotherapy regimen, and the objective response rate in all patients was $13.1 \%$ for pembrolizumab and $6.7 \%$ for the cytotoxic chemotherapy regimen $(\mathrm{p}=0.0037)$ [38]. On the basis of the results of the KEYNOTE-180 Phase II trial and the KEYNOTE-181 Phase III trial, the FDA approved pembrolizumab as second-line chemotherapy for advanced or metastatic ESCC patients with PD-L1 CPS $\geq 10$ on July 30, 2019.

In addition to these trials of previously treated EC patients, a Phase III KEYNOTE-590 trial to compare pembrolizumab plus CF with CF as first-line chemotherapy for untreated patients with metastatic or recurrent EC and esophagogastric junction cancer (NCT03189719) [39] is ongoing Table 1.

In addition to nivolumab and pembrolizumab, camrelizumab is an anti-PD-1 antibody that was developed for metastatic or recurrent ESCC in China. The randomized Phase III ESCORT trial that compared camrelizumab with investigator's choice regimen (docetaxel or irinotecan) as second-line chemotherapy showed superior OS (8.3 vs 6.2 months; HR: 0.71 ; 95\% CI: $0.57-0.87 ; \mathrm{p}=0.001$ ). In the camrelizumab arm, the frequent serious treatment-related adverse events were anemia $(3 \%)$ and hepatic function abnormal $(2 \%)$, and there were seven deaths related to nivolumab (three unknown causes, one enterocolitis, one hepatic function abnormal, one pneumonitis and one myocarditis) [40]. Tislelizumab is anotheranti-PD-1 antibody that was developed for metastatic or recurrent ESCC. The Phase III RATIONALE-306 trial comparing tislelizumab monotherapy with chemotherapy (taxane or irinotecan) as second-line chemotherapy for patients with metastatic or recurrent ESCC (NCT03430843) and a RATIONALE-302 Phase III trial to compare tislelizumab plus chemotherapy (5-FU + platinum or paclitaxel + platinum) with chemotherapy as first-line chemotherapy for untreated patients with metastatic or recurrent ESCC (NCT03783442) are ongoing Table 1.

\section{Development of ICI treatment for unresectable locally advanced EC}

The standard treatment for patients with unresectable locally advanced EC is chemoradiotherapy. Regarding treatment for lung cancer, a Phase III PACIFIC trial comparing chemoradiotherapy followed by durvalumab (anti-PD-L1 antibody) with chemoradiotherapy followed by placebo for patients with unresectable locally advanced non-small-cell lung cancer (NSCLC) was conducted. The primary endpoints were PFS and OS in all patients. All 713 patients were randomly assigned 2:1 to durvalumab or placebo. Chemoradiotherapy followed by durvalumab significantly improved PFS (16.8 vs 5.6 months; HR: $0.52 ; 95 \%$ CI: $0.42-0.65$, p < 0.001 ) compared with chemoradiotherapy followed by placebo [41]. In addition, durvalumab showed superior OS (not reached vs 28.7 months, HR: 0.68 ; 99.73\% CI: $0.47-0.997 ; \mathrm{p}=0.0025)$ compared with placebo in all patients with unresectable locally advanced NSCLC [42].

On the basis of these remarkable results, the development of similar treatment strategies consisting of definitive chemoradiotherapy followed by ICI is in progress for unresectable locally advanced EC. For locally advanced ESCC patients, a Phase II TENERGY trial to evaluate the efficacy and safety of definitive chemoradiotherapy followed by sequential atezolizumab (anti-PD-L1 antibody) monotherapy (UMIN000034373) [43] and a Phase II NOBEL trial to evaluate the efficacy and safety of CF-based definitive chemoradiotherapy plus nivolumab followed by sequential nivolumab monotherapy (UMIN000035889) are ongoing. For inoperable locally advanced EC patients, the Phase II CRUCIAL trial to evaluate efficacy and safety of FOLFOX-based definitive chemoradiotherapy plus nivolumab followed by sequential nivolumab monotherapy and chemoradiotherapy plus nivolumab and ipilimumab followed by sequential nivolumab and ipilimumab (NCT03437200) and a Phase III KEYNOTE-975 trial to evaluate efficacy and safety of CF- or FOLFOX-based chemoradiotherapy plus concurrent pembrolizumab monotherapy (NCT04210115) are ongoing Table 2.

\section{Development of ICI treatment for resectable locally advanced EC}

For resectable locally advanced EC patients, preoperative chemoradiotherapy consisting of chemotherapy followed by surgery is the standard strategy based on the CROSS trial and the JCOG9907 trial [17,19]. To improve clinical outcomes, new treatments consisting of ICI, and these standard treatments are being developed. 


\begin{tabular}{|c|c|c|c|c|c|c|c|}
\hline Clinical trial & Phase & Histology & Patients (n) & Study treatment & pCR (\%) & OS (months) & Ref. \\
\hline \multicolumn{8}{|l|}{ For unresectable EC } \\
\hline TENERGY & II & ESCC & Recruiting & $\mathrm{dCRT}+$ sequential atezolizumab & NA & NA & [43] \\
\hline NOBEL & II & ESCC & Recruiting & $\mathrm{dCRT}+$ concurrent and sequential nivolumab & NA & NA & \\
\hline CRUCIAL & II & ESCC, EAC & Recruiting & $\begin{array}{l}\mathrm{dCRT}+\text { concurrent and sequential nivolumab or } \\
\text { nivolumab and ipilimumab }\end{array}$ & NA & NA & \\
\hline KEYNOTE-975 & III & ESCC, EAC & Recruiting & $\mathrm{dCRT} \pm$ concurrent and sequential pembrolizumab & NA & NA & \\
\hline \multicolumn{8}{|l|}{ For resectable EC } \\
\hline NCT02844075 & II & ESCC & 28 & $\begin{array}{l}\text { Preoperative CRT+ pembrolizumab + postoperative } \\
\text { pembrolizumab }\end{array}$ & 23.1 & Not reached & [45] \\
\hline PERFECT & II & EAC & 40 & Preoperative CRT + atezolizumab & 37 & Not reached & [46] \\
\hline NCT03490292 & $1 / 11$ & EAC & 7 & $\begin{array}{l}\text { Preoperative CRT + avelumab + postoperative } \\
\text { avelumab }\end{array}$ & 43 & NA & [47] \\
\hline NCT03044613 & 1 & ESCC, EAC & 16 & $\begin{array}{l}\text { Induction nivolumab + preoperative CRT + concurrent } \\
\text { nivolumab (Arm A) }\end{array}$ & 31.3 & NA & [44] \\
\hline CheckMate-577 & III & ESCC, EAC & Before analysis & Preoperative CRT \pm postoperative nivolumab & NA & NA & \\
\hline FRONTIER JCOG1804E & 1 & ESCC & Recruiting & $\begin{array}{l}\text { Preoperative } \mathrm{CF}+\text { nivolumab or preoperative } \\
\mathrm{DCF}+\text { nivolumab }\end{array}$ & NA & NA & [51] \\
\hline
\end{tabular}

CF: Cisplatin plus 5-FU; CRT: Chemoradiotherapy; DCF: Docetaxel plus cisplatin plus 5-FU; dCRT: Definitive chemoradiotherapy; EAC: Esophageal adenocarcinoma; EC: Esophageal cancer; ESCC: Esophageal squamous cell carcinoma; NA: Not applicable; OS: Overall survival; pCR: Pathological complete response rate; PFS: Progression-free survival.

Some Phase I and Phase II trials for patients with resectable locally advanced EC have been reported. A Phase I trial to evaluate safety and efficacy of induction nivolumab and preoperative CRT plus nivolumab followed by surgery for patients with resectable EC or gastroesophageal cancer (clinical stage [c-stage] II/III) was conducted. Of 16 eligible patients, adverse events related to any treatment occurred in 12 patients $(75.0 \%)$ and 4 patients $(25.0 \%)$ showed grade 3 adverse events (dyspnea and upper respiratory tract infection, transaminitis, rash). Fourteen patients $(87.5 \%)$ received all planned doses of nivolumab. The pathological complete response rate was $28.6 \%(4 / 14)$ in EAC patients and $50.0 \%(1 / 2)$ in ESCC patients [44]. A Phase II trial to evaluate efficacy and safety of preoperative CRT plus pembrolizumab followed by surgery and postoperative pembrolizumab monotherapy for patients with resectable ESCC (cT1N1-2M0, cT2-4aN0-2M0) was conducted. The pathological complete response rate as the primary endpoint was $23.1 \%(6 / 26)$. However, of 28 eligible patients, three cases of death were reported during the preoperative period (massive hematemesis) and after surgery (acute lung injury) [45]. A Phase II PERFECT trial to evaluate efficacy and safety of preoperative CRT plus atezolizumab followed by surgery for patients with resectable EAC was conducted. The completion ratio (i.e., patients who completed the planned study treatment including atezolizumab/all eligible patients) as the primary endpoint was $75.0 \%(30 / 40)$, and the pathological complete response (Mandard score 1) was 37\% (11/30). In the immune-related safety analysis, two patients (5\%) with grade 3 rash, two patients (5\%) with grade 2 colitis and two patients (5\%) with thyroiditis were reported [46]. A Phase I/II trial to evaluate efficacy and safety of preoperative CRT plus avelumab (anti-PD-L1 antibody) followed by surgery and postoperative avelumab monotherapy for patients with resectable locally advanced EC (cT1N1M0, T2-3N0-2M0) was conducted. The pathological complete response was $43 \%(3 / 7)$. In the immune-related safety analysis, one patient (5\%) with grade 2 hypothyroidism was reported [47].

Previous trials of preoperative chemoradiotherapy followed by surgery for resectable locally advanced EC showed the pathological complete response rate was 28 to $42 \%$ [18,48]. However, it is still unclear whether preoperative chemoradiotherapy plus ICI show survival benefits because these Phase I/II trials only showed short-term efficacy and safety within small number of patients.

The Phase III CheckMate-577 trial to compare nivolumab with placebo as a postoperative treatment after preoperative chemoradiotherapy followed by surgery for resectable EC patients (NCT02743494) is ongoing Table 2.

In addition, a preclinical study to assess perioperative ICI treatment for mice with breast cancer reported the superior survival time of preoperative ICI monotherapy compared with the postoperative setting [49], and a Phase I trial to assess safety and efficacy of preoperative nivolumab monotherapy for resectable NSCLC patients showed the major pathological response ( $\leq 10 \%$ viable tumor cells) was $43 \%$ without increasing the incidence of adverse events [50]. Based on these data, a Phase I JCOG1804E; FRONTiER trial to evaluate the safety and 
efficacy of nivolumab plus CF or nivolumab plus docetaxel and CF for resectable locally advanced ESCC patients (NCT03914443) is ongoing [51] (Table 2).

Therefore, to evaluate the most promising combination therapy with ICI, the long-term results of preoperative chemoradiotherapy plus ICI and those of preoperative chemotherapy plus ICI and preoperative chemoradiotherapy plus postoperative ICI are warranted.

\section{Future perspective}

The development of ICIs is being initiated for various settings of EC in the future; however, differences in evidence between nivolumab and pembrolizumab that can be used at present are being examined.

Nivolumab is a human monoclonal antibody that targets PD-1. The ATTRACTION-3 Phase III trial [35] showed superiority of nivolumab for the second-line treatment of all ESCC patients regardless of PD-L1 expression in contrast to the KEYNOTE-181 Phase III trial [38].

Pembrolizumab is one of the few anticancer drugs that has shown superiority in a Phase III trial for the treatment of EC. However, the superiority for OS compared with cytotoxic agents was only demonstrated in EC patients with PD-L1 CPS $\geq 10$, and superiority was not demonstrated in ESCC patients or all EC patients including EAC patients [38]. The FDA has approved pembrolizumab only for ESCC patients with PD-L1 CPS $\geq 10$.

Therefore, when considering the use of an immune checkpoint inhibitor (ICI) as a salvage-line chemotherapy for ESCC patients, nivolumab, which does not require the immunohistochemical evaluation of PD-L1 expression, might be used more frequently than pembrolizumab.

For metastatic or recurrent ESCC patients, ICIs are standard treatment for second-line chemotherapy based on the results of the ATTRACTION-3 [35] and KEYNOTE-181 [38] trials. Some Phase III trials evaluating the efficacy and safety of combination therapy of cytotoxic drugs and ICI as first-line treatment are ongoing, and ICIcontaining treatment might be the standard first-line therapy depending on these results. However, for metastatic or recurrent EAC patients, there is little evidence for the efficacy of ICI. Therefore, it is necessary to identify predicting biomarkers and develop new combination therapies.

For locally advanced EC patients, new treatments consisting of chemotherapy or chemoradiotherapy plus ICI are just beginning to be developed, and evidence for their efficacy and safety are limited. Therefore, the results of ongoing trials will be of interest to determine whether the efficacy of ICI is different between ESCC and EAC and whether treatment selection by biomarkers is useful.

\section{Executive summary}

Metastatic or recurrent EC

- Nivolumab monotherapy showed efficacy for patients with metastatic or recurrent esophageal squamous cell carcinoma (ESCC) as second-line treatment. Pembrolizumab monotherapy showed efficacy for metastatic or recurrent esophageal cancer (EC) patients with CPS $\geq 10 \%$.

- Currently, Phase III trials to evaluate the efficacy of chemotherapy with nivolumab or pembrolizumab for first-line treatment (CheckMate-648, KEYNOTE-590) and Phase III trials to evaluate the efficacy of chemotherapy including tislelizumab in first-line and second-line treatments (NCT03783442, NCT03430843) are ongoing.

Unresectable locally advanced EC

- On the basis of the results of the PACIFIC trial, some Phase II trials and the KEYNOTE-975 Phase III trial (NCT04210115) to evaluate efficacy and safety of immune checkpoint inhibitors (ICI)-containing chemoradiotherapy strategy for mainly ESCC patients are ongoing.

Resectable locally advanced EC

- Some Phase II trials to assess efficacy and safety of immune checkpoint inhibitor (ICI)-containing preoperative chemoradiotherapy with/without postoperative $\mathrm{ICl}$ showed limited efficacy in their short-term results. The results of a trial to evaluate safety and efficacy of postoperative ICI monotherapy (CheckMate-577) and ICl-containing preoperative chemotherapy (JCOG1804E, FRONTiER) are awaited. 


\section{Open access}

This work is licensed under the Attribution-NonCommercial-NoDerivatives 4.0 Unported License. To view a copy of this license, visit http://creativecommons.org/licenses/by-nc-nd/4.0/

\section{References}

Papers of special note have been highlighted as: $\bullet \bullet$ of considerable interest

1. WHO. GLOBOCAN 2018 estimated cancer incidence, mortality and prevalence worldwide (2018). http://globocan.iarc.fr/

2. Thrift AP. The epidemic of oesophageal carcinoma: where are we now? Cancer epidemiol. 41, 88-95 (2016).

3. Rustgi AK, El-Serag HB. Esophageal carcinoma. N. Engl. J. Med. 371, 2499-2509 (2014).

4. El-Serag HB, Hashmi A, Garcia J et al. Visceral abdominal obesity measured by CT scan is associated with an increased risk of Barrett's oesophagus: a case-control study. Gut. 63, 220-229 (2014).

5. Njei B, McCarty TR, Birk JW. Trends in esophageal cancer survival in United States adults from 1973 to 2009: a SEER database analysis. J. Gastroenterol Hepatol. 31(6), 1141-1146 (2016).

6. NCCN guidelines ver. 32020 Esophageal and esophagogastric junction cancers (2020). www.nccn.org/professionals/physician_gls/pdf/esophageal.pdf

7. Lordick F, Mariette $\mathrm{C}$, Haustermans $\mathrm{K}$ et al. Oesophageal cancer: ESMO Clinical Practice Guidelines for diagnosis, treatment and follow-up. Ann. Oncol. 27(suppl. 5), v50-v57 (2016).

8. Kitagawa Y, Uno T, Oyama T et al. Esophageal cancer practice guidelines 2017 edited by the Japan esophageal society: part 2. Esophagus. 16(1), 25-43 (2019).

9. Iizuka T, Kakegawa T, Ide H et al. Phase II evaluation of cisplatin and 5-fluorouracil in advanced squamous cell carcinoma of the esophagus: a Japanese Esophageal Oncology Group Trial. Jpn J. Clin. Oncol. 22(3), 172-176 (1992).

10. Hayashi K, Ando N, Watanabe $\mathrm{H}$ et al. Phase II evaluation of protracted infusion of cisplatin and 5-fluorouracil in advanced squamous cell carcinoma of the esophagus: a Japan Esophageal Oncology Group (JEOG) Trial (JCOG9407). Jpn J. Clin. Oncol. 31(9), 419-23 (2001).

11. Kato K, Tahara M, Hironaka S et al. A Phase II study of paclitaxel by weekly 1-h infusion for advanced or recurrent esophageal cancer in patients who had previously received platinum-based chemotherapy. Cancer Chemother. Pharmacol. 67(6), 1265-1272 (2011).

12. Muro K, Hamaguchi T, Ohtsu A et al. A Phase II study of single-agent docetaxel in patients with metastatic esophageal cancer. Ann Oncol. 15(6), 955-959 (2004).

13. Janowitz T, Thuss-Patience P, Marshall A et al. Chemotherapy vs supportive care alone for relapsed gastric, gastroesophageal junction, and oesophageal adenocarcinoma: a meta-analysis of patient-level data. Br. J. Cancer. 114(4), 381-387 (2016).

14. Minsky BD, Pajak TK, Ginsberg RJ et al. INT 0123 (Radiation Therapy Oncology Group 94-05) Phase III trial of combined-modality therapy for esophageal cancer: high-dose versus standard-dose radiation therapy. J. Clin. Oncol. 20(5), 1167-1174 (2002).

15. Ishida K, Ando N, Yamamoto S et al. Phase II study of cisplatin and 5-fluorouracil with concurrent radiotherapy in advanced squamous cell carcinoma of the esophagus: a Japan Esophageal Oncology Group (JEOG)/Japan Clinical Oncology Group trial (JCOG9516). Jpn J. Clin. Oncol. 34(10), 615-619 (2004).

16. Conroy T, Galais MP, Raoul JL et al. Definitive chemoradiotherapy with FOLFOX versus fluorouracil and cisplatin in patients with oesophageal cancer (PRODIGE5/ACCORD17): final results of a randomised, Phase 2/3 trial. Lancet Oncol. 15(3), 305-314 (2014).

17. Ando N, Kato $\mathrm{H}$, Igaki $\mathrm{H}$ et al. A randomized trial of postoperative adjuvant chemotherapy with cisplatin and 5-fluorouracil versus neoadjuvant chemotherapy for clinical stage II/III squamous cell carcinoma of the thoracic esophagus (JCOG 9907). Ann. Surg. Oncol. 19(1), 68-74 (2012).

18. Klevebro F, von Döbeln G Alexandersson, Wang N et al. A randomized clinical trial of neoadjuvant chemotherapy versus neoadjuvant chemoradiotherapy for cancer of the oesophagus or gastro-oesophageal junction. Ann. Oncol. 27(4), 660-667 (2016).

19. van Hagen P, Hulshof MC, van Lanschot JJ et al. Preoperative chemoradiotherapy for esophageal or junctional cancer. N. Engl. J. Med. 366, 2074-2084 (2012).

20. Robert C, Schachter J, Long GV et al. Pembrolizumab versus ipilimumab in advanced melanoma. N. Engl. J. Med. 372(26), 2521-2532 (2015).

21. Robert C, Ribas A, Schachter J et al. Pembrolizumab versus ipilimumab in advanced melanoma (KEYNOTE-006): post-hoc 5-year results from an open-label, multicentre, randomised, controlled, Phase 3 study. Lancet Oncol. 20(9), 1239-1251 (2019).

22. Reck M, Rodríguez-Abreu D, Robinson AG et al. Pembrolizumab versus chemotherapy for PD-L1-positive non-small-cell lung cancer. N. Engl. J. Med. 375(19), 1823-1833 (2016).

23. Gandhi L, Rodríguez-Abreu D, Gadgeel S et al. Pembrolizumab plus chemotherapy in metastatic non-small-cell lung cancer. $N$. Engl. J. Med. 378(22), 2078-2092 (2018).

24. Chen R, Zinzani PL, Fanale MA et al. Phase II study of the efficacy and safety of pembrolizumab for relapsed/refractory classic Hodgkin lymphoma. J. Clin. Oncol. 35(19), 2125-2132 (2017). 
25. Balar AV, Castellano D, O’Donnell PH et al. First-line pembrolizumab in cisplatin-ineligible patients with locally advanced and unresectable or metastatic urothelial cancer (KEYNOTE-052): a multicentre, single-arm, Phase 2 study. Lancet Oncol. 18(11), 1483-1492 (2017).

26. Bellmunt J, de Wit R, Vaughn DJ et al. Pembrolizumab as second-line therapy for advanced urothelial carcinoma. N. Engl. J. Med. 376(11), 1015-1026 (2017).

27. Cohen EEW, Soulières D, Le Tourneau C et al. Pembrolizumab versus methotrexate, docetaxel, or cetuximab for recurrent or metastatic head-and-neck squamous cell carcinoma (KEYNOTE-040): a randomised, open-label, Phase 3 study. Lancet 393(10167), 156-167 (2019).

28. Burtness B, Harrington KJ, Greil R et al. Pembrolizumab alone or with chemotherapy versus cetuximab with chemotherapy for recurrent or metastatic squamous cell carcinoma of the head and neck (KEYNOTE-048): a randomised, open-label, Phase 3 study. Lancet 394(10212), 1915-1928 (2019).

29. Schellens H.M Jan, Marabelle Aurelien, Zeigenfuss Susan et al. Pembrolizumab for previously treated advanced cervical squamous cell cancer: preliminary results from the Phase 2 KEYNOTE-158 study. J. Clin. Oncol. 35(Suppl.), abstr 5514 (2017).

30. Fuchs CS, Doi T, Jang RW et al. Safety and efficacy of pembrolizumab monotherapy in patients with previously treated advanced gastric and gastroesophageal junction cancer: Phase 2 clinical KEYNOTE-059 trial. JAMA Oncol. 4(5), e180013 (2018).

31. Zhu AX, Finn RS, Edeline J et al. Pembrolizumab in patients with advanced hepatocellular carcinoma previously treated with sorafenib (KEYNOTE-224): a non-randomised, open-label Phase 2 trial. Lancet Oncol. 19(7), 940-952 (2018).

32. Le DT, Uram JN, Wang H et al. PD-1 blockade in tumors with mismatch-repair deficiency. N. Engl. J. Med. 372(26), 2509-2520 (2015).

33. Brahmer JR, Drake CG, Woller I et al. Phase I study of single-agent anti-programmed death-1 (MDX-1106) in refractory solid tumors: safety, clinical activity, pharmacodynamics, and immunologic correlates. J. Clin. Oncol. 28, 3167-75 (2010).

34. Kudo T, Hamamoto Y, Kato K et al. Nivolumab treatment for oesophageal squamous-cell carcinoma: an open-label, multicentre, Phase 2 trial. Lancet Oncol. 18, 631-639 (2017).

-• Multicenter, Phase II trial reporting the efficacy and safety of nivolumab monotherapy for heavy pretreated metastatic esophageal squamous cell carcinoma patients.

35. Kato K, Cho BC, Takahashi M et al. Nivolumab versus chemotherapy in patients with advanced oesophageal squamous cell carcinoma refractory or intolerant to one prior chemotherapy (ATTRACTION-3): a randomised, open-label, Phase 3 trial. Lancet Oncol. 20(11), 1506-1517 (2019).

-• Global, multicenter, open-label, Phase III trial (ATTRACTION-3) comparing the efficacy and safety of nivolumab monotherapy and taxane for unresectable advanced or recurrent esophageal squamous cell carcinoma patients who were refractory or intolerant to one previous chemotherapy.

36. Janjigian Y Yelena, Bendell Johanna, Calvo Emiliano et al. CheckMate-032 study: efficacy and safety of nivolumab and nivolumab plus ipilimumab in patients with metastatic esophagogastric cancer. J. Clin. Oncol. 36(28), 2836-2844 (2018).

37. Shah MA, Kojima T, Hochhauser D et al. Efficacy and safety of pembrolizumab for heavily pretreated patients with advanced, metastatic adenocarcinoma or squamous cell carcinoma of the esophagus: the Phase 2 KEYNOTE-180 study. JAMA Oncol. 5(4), 546-550 (2019).

-. Multicenter, single-arm, Phase II trial evaluating the efficacy and safety of pembrolizumab monotherapy for treated metastatic esophageal cancer patients.

38. Kojima T, Muro K, Francois E et al. Pembrolizumab versus chemotherapy as second-line therapy for advanced esophageal cancer: Phase III KEYNOTE-181 study. J. Clin. Oncol. 37(Suppl. 4), abstr 2 (2019).

-• Global, multicenter, open-label, Phase III trial (KEYNOTE-181) comparing the efficacy and safety of pembrolizumab monotherapy and taxane or irinotecan for unresectable advanced or recurrent esophageal cancer patients in second-line treatment.

39. Kato K, Shah MA, Enzinger P et al. KEYNOTE-590: Phase III study of first-line chemotherapy with or without pembrolizumab for advanced esophageal cancer. Future Oncol. 15(10), 1057-1066 (2019).

40. Huang Jing, Jianming $\mathrm{Xu}$, Chen Yun $e t$ al. Camrelizumab versus investigator's choice of chemotherapy as second-line therapy for advanced or metastatic oesophageal squamous cell carcinoma (ESCORT): a multicentre, randomised, open-label, Phase 3 study. Lancet Oncol. 21(6), 832-842 (2020).

41. Antonia SJ, Villegas A, Daniel D et al. Durvalumab after chemoradiotherapy in stage III non-small-cell lung cancer. N. Engl. J. Med. 377(20), 1919-1929 (2017).

-• Global, multicenter, placebo-controlled, Phase III trial (PACIFIC) reporting progression-free survival between durvalumab consolidation therapy and placebo after definitive chemoradiotherapy for stage III non-small-cell lung cancer patients.

42. Antonia SJ, Villegas A, Daniel D et al. Overall survival with durvalumab after chemoradiotherapy in stage III NSCLC. N. Engl. J. Med. 379(24), 2342-2350 (2018).

-• Global, multicenter, placebo-controlled, Phase III trial (PACIFIC) reporting overall survival between durvalumab consolidation therapy and placebo after definitive chemoradiotherapy for stage III non-small-cell lung cancer patients. 
43. Bando H, Kotani D, Tsushima T et al. TENERGY: multicenter Phase II study of Atezolizumab monotherapy following definitive Chemoradiotherapy with 5-FU plus Cisplatin in patients with unresectable locally advanced esophageal squamous cell carcinoma. $B M C$ Cancer 20(1), 336 (2020).

44. Kelly RJ, Smith KN, Anagnostou V et al. Neoadjuvant nivolumab plus concurrent chemoradiation in stage II/III esophageal/gastroesophageal junction cancer. J. Clin. Oncol. 37(Suppl.), abstr 142 (2019).

45. Hong HM, Kim RH, Park YS et al. A Phase II trial of preoperative chemoradiotherapy and pembrolizumab for locally advanced esophageal squamous cell carcinoma (ESCC). J. Clin. Oncol. 37(Suppl.), abstr 4027 (2019).

46. van den Ende T, Clercq de C N, van Berge HIM et al. A Phase II feasibility trial of neoadjuvant chemoradiotherapy combined with atezolizumab for resectable esophageal adenocarcinoma: The PERFECT trial. J. Clin. Oncol. 37(Suppl.), abstr 4045 (2019).

47. Uboha VN, Maloney DJ, McCarthy D et al. Safety of neoadjuvant chemoradiation (CRT) in combination with avelumab (A) in the treatment of resectable esophageal and gastroesophageal junction (E/GEJ) cancer. J.Clin. Oncol. 37(Suppl.), abstr 4041 (2019).

48. Hashimoto J, Kato K, Ito Y et al. Phase II feasibility study of preoperative concurrent chemoradiotherapy with cisplatin plus 5-fluorouracil and elective lymph node irradiation for clinical stage II/III esophageal squamous cell carcinoma. Int. J. Clin. Oncol. 24, 60-67 (2019).

49. Liu J, Blake SJ, Yong MC et al. Improved efficacy of neoadjuvant immunotherapy to eradicate metastatic disease. Cancer Discov. 6, 1382-1399 (2016).

50. Forde PM, Chaft JE, Smith KN et al. Neoadjuvant PD-1 blockade in resectable lung cancer. N. Eng. J. Med. 378, 1976-1986 (2018).

51. Yamamoto S, Kato K, Daiko H et al. Feasibility study of nivolumab as neoadjuvant chemotherapy for locally esophageal carcinoma: FRONTiER (JCOG1804E). Future Oncol. 16(19), 1351-1357 (2020). 